This is a self-archived version of an original article. This version may differ from the original in pagination and typographic details.

Author(s): Neittaanmäki, P.; Nokka, M.; Repin, S.

Title: Estimates of the Distance to Exact Solutions of the Stokes Problem with Slip and Leak Boundary Conditions

Year: 2019

Version: Accepted version (Final draft)

Copyright: @ 2019 Springer Science+Business Media, LLC

Rights: In Copyright

Rights url: http://rightsstatements.org/page/InC/1.0/?language=en

Please cite the original version:

Neittaanmäki, P., Nokka, M., \& Repin, S. (2019). Estimates of the Distance to Exact Solutions of the Stokes Problem with Slip and Leak Boundary Conditions. Journal of Mathematical Sciences, 242(2), 280-298. https://doi.org/10.1007/s10958-019-04477-6 
Journal of Mathematical Sciences, Vol. 000, No. 0, 000, 2019

\title{
ESTIMATES OF THE DISTANCE TO EXACT SOLUTIONS OF THE STOKES PROBLEM WITH SLIP AND LEAK BOUNDARY CONDITIONS
}

\author{
P. Neittaanmäki \\ Department of mathematical information technology \\ University of Jyväskylä, FI-40014, Finland \\ pekka.neittaanmaki@jyu.fi
}

M. Nokka -2pt Department of mathematical information technology

University of Jyväskylä, FI-40014, Finland

marjaana.nokka@jyu.fi

\section{S. Repin*}

V. A. Steklov Institute of Mathematics RAS

27, Fontanka, St. Petersburg 191011, Russia

Peter the Great St. Petersburg Polytechnic University

29, Polytekhnicheskaya St., St. Petersburg 195251, Russia

repin@pdmi.ras.ru

UDC 519.6

We deduce a posteriori error estimates of functional type for the stationary Stokes problem with slip and leak boundary conditions. The derived error majorants do not contain mesh dependent constants and are valid for a wide class of energy admissible approximations that satisfy the Dirichlet boundary condition on a part of the boundary. Different forms of error majorants contain global constants associated with Poincaré type inequalities or the stability $(L B B)$ condition for the Stokes problem or constants associated with subdomains (if a domain decomposition is applied). It is proved that the majorants are guaranteed and vanish if and only if the functions entering them coincide with the respective exact solutions. Bibliography: 38 titles. Illustrations: 1 figure.

Dedicated to the jubilee of dear Nina Nikolaevna Uraltseva

\section{Introduction}

Mathematical models of viscous incompressible fluids with nonlinear boundary conditions were introduced and studied by Fujita [1]-[3], who proved the existence and uniqueness of weak (generalized) solutions to the Stokes problem under leak or slip boundary conditions. These models are often used in various applied problems (for example, in analysis of oil flow beneath

\footnotetext{
* To whom the correspondence should be addressed.
}

Translated from Problemy Matematicheskogo Analiza 00, 2019, pp. 00-00.

1072-3374/19/2393-0248 (C) 2019 Springer Science+Business Media, LLC 
or over sand layers $[4,5]$, simulation of blood flow, modeling of avalanches of water and rocks). Numerical methods for this class of nonlinear problems are well developed (cf., for example, [6]-[14] and many other publications cited therein).

In this paper, we address a different problem, which arises if a numerical solution has been already found and we need to estimate the distance between the exact solution and this approximation. For the considered class of problems we deduce estimates (hereafter called error majorants) that solve this problem. A majorant includes only known data and known functions (approximations of the velocity, stress, and pressure fields). Also, they contain global constants associated with certain functional inequalities (Friedrichs, Poincaré, LBB, Korn) related to the problem in question. Estimates of these constants can be found either computationally (by well known numerical methods) or analytically. The latter way is based on decomposition of $\Omega$ into a collection of "simple" subdomains, for which the respective constants (or sharp estimates of them) are known. Hence a majorant is a computable nonnegative functional that provides an upper bound of the error measured in terms of the natural energy norm. We prove that it vanishes if and only if an approximate solution substituted into the majorant coincides with the exact solution. It should be outlined that the majorants are derived by purely functional methods without attracting specific features of approximations (for example, Galerkin orthogonality) or a numerical method by which they were obtained. Therefore, they are valid for any approximation in the energy space satisfying the main boundary conditions.

Our analysis is based on the theory of functional a posteriori error estimates (for a systematic exposition of this theory in the context of viscous incompressible fluids we refer to [15]-[17]). For some problems with nonlinear boundary conditions estimates of this type have been earlier obtained in $[18,19]$ by methods of duality theory and convex analysis (which differs from the method used in this paper). In [20], the estimates were used for analysis of approximations generated by the Uzawa method.

1.1. Notation and basic equations. Throughout the paper, $\Omega$ denotes a connected bounded domain in $\mathbb{R}^{d}(d=2,3)$ with Lipschitz continuous boundary $\Gamma$, which consists of two disjoint measurable parts $\Gamma_{1}$ and $\Gamma_{2}\left(\operatorname{meas}_{d-1} \Gamma_{1}>0\right)$. We denote by $\{\varphi\}_{\Omega}$ the mean value of a summable function $\varphi$ in $\Omega$, and $\widetilde{L}_{2}(\Omega)$ coincides with $L^{2}(\Omega)$ if $\Gamma \neq \Gamma_{1}$ and is defined as the subspace of $L^{2}(\Omega)$ containing functions with zero mean values in the case of full Dirichlet boundary conditions. Next, $V:=H^{1}\left(\Omega, \mathbb{R}^{d}\right)$ is the Sobolev space of vector-valued functions having square summable derivatives of the first order and $V_{0}$ is a subspace of $V$ containing functions vanishing on $\Gamma_{1}$. We denote by $\mathbb{S}(\Omega)$ a subspace of $V$ containing solenoidal (divergence free) functions. Analogously, $\mathbb{S}_{0}(\Omega)$ denotes the subspace of $\mathbb{S}(\Omega)$ containing functions vanishing on $\Gamma_{1}$.

Also, we use the space of tensor-valued functions $\Sigma(\Omega):=L^{2}\left(\Omega, \mathbb{M}^{d \times d}\right)$, where $\mathbb{M}^{d \times d}$ is the space of real $d \times d$-matrices (tensors) with the unit element $\mathbb{I}$ and scalar product denoted by two dots :, $\tau n$ denotes the normal component (vector) of the tensor $\tau$. Since no confusion may arise, we use one common notation $\|\cdot\|$ for the $L^{2}$-norms of scalar, vector, and tensor-valued functions, for example,

$$
\|\tau\|^{2}:=\int_{\Omega}|\tau|^{2} d x=\int_{\Omega} \tau: \tau d x .
$$

This norm is associated with the corresponding inner product ( , ). If it is associated with a set $\omega$ that differs from $\Omega$, then the norm is written as $\|\cdot\|_{\omega}$. We denote by div and Div the 
divergence operators for the vector and tensor fields respectively. Henceforth, we use the bilinear form $a: V \times V \rightarrow \mathbb{R}$

$$
a(u, v):=\int_{\Omega} \nu \varepsilon(u): \varepsilon(v) d x,
$$

where

$$
\varepsilon(u)=\frac{1}{2}\left(\frac{\partial u_{i}}{\partial x_{j}}+\frac{\partial u_{j}}{\partial x_{i}}\right)
$$

is the symmetric part of the gradient tensor and $\nu$ is a function (viscosity) bounded from below and above by positive constants $\underline{\nu}$ and $\bar{\nu}$. For the sake of convenience, we additionally introduce the norms

$$
\begin{aligned}
& \|\eta\|_{\nu}^{2}:=\int_{\Omega} \nu \tau: \tau d x, \\
& \|\eta\|_{1 / \nu}^{2}:=\int_{\Omega} \nu^{-1} \tau: \tau d x,
\end{aligned}
$$

which are equivalent to the norm of $\Sigma(\Omega)$.

1.2. Estimates of the distance to the exact solution of the Stokes problem. We begin with a short overview of previous results related to the derivation of estimates that control the distance between the exact solution of the Stokes problem and a vector-valued function considered as an approximation of it. The classical Stokes problem with the Dirichlet-Neumann boundary conditions consists of finding a velocity field $u \in V$ and a pressure field $p \in \widetilde{L}^{2}(\Omega)$ such that

$$
\begin{aligned}
& -\operatorname{Div} \eta+\nabla p=f \quad \text { in } \Omega, \\
& \operatorname{div} u=0 \quad \text { in } \Omega, \\
& \eta=\nu \varepsilon(u) \quad \text { in } \Omega, \\
& u=u_{D} \quad \text { on } \Gamma_{1}, \\
& \sigma n=F \quad \text { on } \Gamma_{2},
\end{aligned}
$$

where $f \in L^{2}\left(\Omega, \mathbb{R}^{d}\right), F \in L^{2}\left(\Gamma_{2}, \mathbb{R}^{d}\right), n$ denotes the unit outward normal vector to the boundary, $u_{D}$ is a given divergence free function that defines the boundary condition on $\Gamma_{1}$ (in the sense that the trace of $u$ coincides with the trace of $\left.u_{D}\right)$, and $\varepsilon(u)$ is connected with the stress tensor $\sigma$ by the relation $\sigma=-p \mathbb{I}+\nu \varepsilon(u)$.

It is well known (cf., for example, [21, 22]) that the problem (1.1)-(1.5) possesses a unique generalized solution and the respective function $u$ satisfies the integral identity

$$
a(u, w)=\int_{\Omega} f \cdot w d x+\int_{\Gamma_{2}} F w d s \quad \forall w \in \mathbb{S}_{0}(\Omega) .
$$

Let $v \in V_{0}+u_{D}$ be a vector-valued function considered as an approximation of (1.6). Our goal is to deduce a fully computable majorant of the distance between $u$ and $v$ measured in terms of the natural energy norm. To be of practical relevance, the majorant must be continuous with respect 
to $v$, vanish only if $v$ coincides with the exact solution $u$ and do not generate significant gaps between the error and the estimate. For the Stokes problem the respective estimates have been derived in $[16,15]$. These estimates are not restricted to the case of divergence free fields. It is only required that $v$ satisfies the condition $\{\operatorname{div} v\}_{\Omega}=0$ (if $v$ does not satisfy this integral type condition, then is easy to fulfil it by a suitable correction of the velocity field). Let $q \in \widetilde{L}^{2}(\Omega)$ be an approximation of the pressure $p$ and $\tau \in \Sigma(\Omega)$ be an approximation of the exact stress $\sigma$. In the simplest case, the majorant of the distance between $v$ and $u$ has the form:

$$
\begin{aligned}
\|\varepsilon(u-v)\|_{\nu} & \leqslant M(v, q, \tau):=\|\nu \varepsilon(v)-\tau-\mathbb{I} q\| \\
& +C_{\Omega}\|\operatorname{Div} \tau+f\|+C_{\Gamma}\|\tau n-F\|+2 \nu c_{\Omega}\|\operatorname{div} v\| .
\end{aligned}
$$

Here, $\tau$ is any tensor-valued function in the set

$$
H_{\Gamma_{2}}(\Omega, \operatorname{Div}):=\left\{\tau \in \Sigma \mid \operatorname{Div} \tau \in L^{2}\left(\Omega, \mathbb{R}^{d}\right), \tau n \in L^{2}\left(\Gamma_{2}, \mathbb{R}^{d}\right)\right\},
$$

$C_{\Omega}$ and $C_{\Gamma}$ are constants in the inequalities

$$
\|w\| \leqslant C_{\Omega}\|\varepsilon(w)\|, \quad\|w\|_{\Gamma_{2}} \leqslant C_{\Gamma}\|\varepsilon(w)\|,
$$

which hold for any $w \in V_{0}, c_{\Omega}$ is the constant coming from the following well known result (often called the "existence of a bounded right inverse operator to the operator div" or the "stability lemma" for the Stokes problem).

Lemma 1.1. For any function $g \in \widetilde{L}_{2}(\Omega)$ there exists a function $v \in V$ vanishing on the boundary and such that $\operatorname{div} v=g$ and

$$
\|\nabla v\| \leqslant c_{\Omega}\|g\|
$$

where $c_{\Omega}$ is a positive constant depending on the shape of $\Omega$ (independent of scaling).

This result (in different forms) has been discovered by several authors. Probably the earliest publication is by Nečas [23] who established it in the form of a weak Poincaré type inequality for functions with gradients in $H^{-1}$. For $d=2$ Lemma 1.1 was established in [24] and for $d \geqslant 2$ in [22]. This result can be also formulated as the inf-sup condition (cf. [25]). In the literature, it is often called the Ladyzhenskaya-Babuška-Brezzi (LBB) condition).

It is worth adding comments concerning the constants entering (1.7) and other similar estimates arising in the other problems. For domains with complicated boundaries the constants $C_{\Omega}, C_{\Gamma}$, and $c_{\Omega}$ may be unknown. In [15], it was shown that using ideas of domain decomposition, difficulties related to $C_{\Omega}$ can be avoided and this constant is replaced by constants $C_{P}\left(\Omega_{i}\right)$ in the Poincaré inequalities related to subdomains $\Omega_{i}$ covering $\Omega$ (for convex $\Omega_{i}$ we know that $C_{P}\left(\Omega_{i}\right) \leqslant \pi^{-1}$ diam $\Omega_{i} ;$ cf. [26]).

It was shown (cf. [17] and some other publications cited therein) that $c_{\Omega}$ can be also replaced by a collection of local constants associated with simple subdomains (this result is based on a modified version of Lemma 1.1). Estimates of the constant $c_{\Omega}$ has been studied by several authors (mostly for the case $d=2$; cf., for example, [27]-[32]). Thus, for relatively simple domains (for example, triangles, rectangles) we have explicit estimates of $c_{\Omega}$ and using decomposition we are able to deduce estimates of the distance to exact solutions of the Stokes and Oseen problem containing local inf-sup constants (associated with subdomains) instead of $c_{\Omega}$ (associated with 
$\Omega$, which may have a complicated form). If the function $v$ is a numerical approximation of $u$ (for example, it is a finite element approximation), then the estimate (1.7) can be considered as an a posteriori estimate of the accuracy. Computational applicability of such type estimates is well studied (for example, cf. an overview in [33, Section 4.2] and the references cited in this book). For the Stokes problem (1.1)-(1.5) (and modifications including rotation and reaction terms), the estimates provide efficient and guaranteed bounds of approximation errors. Moreover, the first term of the majorant $M(v, q, \tau)$ generates a robust indicator of local errors that can be used for mesh adapting procedures. The majorants derived below for problems with slip and leak boundary conditions contain the same terms except one: the nonlinear boundary condition is accounted by the term $D_{j}$, which is a compound functional formed by the boundary functional $j$ and its conjugate $j^{*}$. The goal of this paper is to prove the estimates and to show that they possess the same mathematical properties as the majorants derived for the classical Stokes problem.

The trace constant $C_{\Gamma}$ is excluded from the majorant if $\tau n=F$ on $\Gamma_{2}$ (this condition is easy to fulfil if, for example, $F$ is constant and $\Gamma_{2}$ is piecewise affine). However, this boundary condition (as well as other more general conditions considered below) may be difficult to exactly satisfy if $F$ or/and $\Gamma_{2}$ have complicated shapes. For this reason, we apply a different modus operandi and estimate the boundary integral term by a version of the Poincare inequality (the so-called "sloshing" inequality (2.10)) valid for functions with zero mean traces on the boundary. This method leads to error majorants containing much simpler constants.

Theorems 2.2 and 3.2 present the main results of the paper. They propose fully computable upper bounds of the distance to the exact solutions of problems with slip and leak boundary conditions, respectively.

1.3. Nonlinear boundary conditions. We consider the following nonlinear boundary conditions on $\Gamma_{2}$ :

$$
u_{n}=0, \quad-\varsigma_{t} \in \varkappa \partial\left|u_{t}\right| \quad \text { slip type condition) }
$$

or

$$
u_{t}=0, \quad-\varsigma_{n} \in \varkappa \partial\left|u_{n}\right| \quad \text { leak type condition). }
$$

Here, $\varkappa \geqslant 0$ is a constant, $u_{n}:=u \cdot n, u_{t}:=u-n u_{n}$ (normal and tangential components of the velocity vector). Henceforth, we use standard notation for the tangential and normal components of a tensor:

$$
\tau_{n}:=\tau n, \quad \tau_{t}:=\tau_{n}-n \tau_{n n}, \quad \tau_{n n}:=\tau_{n} \cdot n .
$$

The scalar function $\varsigma_{n}$ and the vector-valued function $\varsigma_{t}$ are defined by the relations (1.11) and (1.12) and represent the normal and tangential components of the stress, respectively.

Certainly, formulas (1.13) are related to the classical statement, and we assume that $u$ possesses necessary differentiability properties up to the boundary. Since

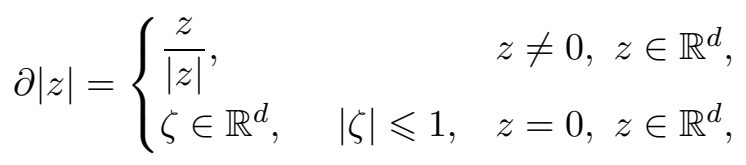

the conditions (1.9) and (1.10) are equivalent to

$$
\left|\varsigma_{t}\right| \leqslant \varkappa, \quad \varsigma_{t} \cdot u_{t}+\varkappa\left|u_{t}\right|=0 \quad \text { on } \Gamma_{2}
$$


or

$$
\left|\varsigma_{n}\right| \leqslant \varkappa, \quad \varsigma_{n} u_{n}+\varkappa\left|u_{n}\right|=0 \quad \text { on } \Gamma_{2}
$$

respectively.

In order to present the generalized statements, we define the sets $\mathbb{S}_{n, 0}$ and $\mathbb{S}_{t, 0}$ that contain the divergence free functions satisfying the main boundary conditions, i.e.,

$$
\begin{aligned}
& \mathbb{S}_{n, 0}:=\left\{v \in \mathbb{S} \mid v=0 \text { on } \Gamma_{1}, v_{n}=0 \text { on } \Gamma_{2}\right\} \\
& \mathbb{S}_{t, 0}:=\left\{v \in \mathbb{S} \mid v=0 \text { on } \Gamma_{1}, v_{t}=0 \text { on } \Gamma_{2}\right\}
\end{aligned}
$$

It is clear that

$$
\begin{aligned}
& \mathbb{S}_{n, 0} \subset V_{n, 0}:=\left\{w \in V \mid w=0 \text { on } \Gamma_{1}, w_{n}=0 \text { on } \Gamma_{2}\right\} \\
& \mathbb{S}_{t, 0} \subset V_{t, 0}:=\left\{w \in V \mid w=0 \text { on } \Gamma_{1}, w_{t}=0 \text { on } \Gamma_{2}\right\} .
\end{aligned}
$$

Now, $\varsigma_{n}$ and $\varsigma_{t}$, are defined in the distributional sense by means of the integral identities (cf. $[1,3])$. For the component $\varsigma_{n}$ we have

$$
\int_{\Gamma_{2}} \varsigma_{n} w_{n} d s=a(u, w)-(p, \operatorname{div} w)-(f, w) \quad \forall w \in V_{t, 0},
$$

where $w_{n}$ denotes the normal component of the trace of $w$ on $\Gamma_{2}$. Analogously,

$$
\int_{\Gamma_{2}} \varsigma_{t} \cdot w_{t} d s=a(u, w)-(p, \operatorname{div} w)-(f, w) \quad \forall w \in V_{n, 0}
$$

If $u$ is sufficiently regular, then integrating by parts and using (1.1)-(1.2), we conclude that

$$
\varsigma_{n}=-p+\nu \varepsilon_{n n}(u), \quad \varsigma_{t}=\nu \varepsilon_{t}(u) .
$$

The generalized solution $u \in \mathbb{S}_{n, 0}+u_{D}$ of the problem with the boundary condition (1.9) satisfies the variational inequality $[1,3]$

$$
a(u, w-u)+\int_{\Gamma_{2}}\left(j\left(w_{t}\right)-j\left(u_{t}\right)\right) d s \geqslant(f, w-u) \quad \forall w \in \mathbb{S}_{n, 0}+u_{D},
$$

where $j(\zeta):=\varkappa|\zeta|$. In the case of (1.10), the generalized solution $u \in \mathbb{S}_{t, 0}+u_{D}$ satisfies

$$
a(u, w-u)+\int_{\Gamma_{2}}\left(j\left(w_{n}\right)-j\left(u_{n}\right)\right) d s \geqslant(f, w-u) \quad \forall w \in \mathbb{S}_{t, 0}+u_{D} .
$$

We note that the above two problems can be formulated as variational problems for convex and coercive functionals. Therefore, the existence and uniqueness of the solutions follow from general theorems of the calculus of variations (cf., for example, [34, 35]). 


\section{Slip Boundary Condition}

2.1. Estimates for approximations in $\mathbb{S}_{n, 0}+u_{D}$. Let $v$ be a divergence free vector-valued function in $\mathbb{S}_{n, 0}+u_{D}$ considered as an approximation of $u$. We rewrite (1.14) in the form

$$
a(u-v, v-u)+\int_{\Gamma_{2}}\left(j\left(v_{t}\right)-j\left(u_{t}\right)\right) d s \geqslant(f, v-u)-a(v, v-u)
$$

which implies the estimate

$$
\begin{aligned}
\|\varepsilon(u-v)\|_{\nu}^{2} & \leqslant \int_{\Omega}(\nu \varepsilon(v)-\tau-\mathbb{I} q): \varepsilon(v-u) d x \\
& +\int_{\Gamma_{2}}\left(j\left(v_{t}\right)-j\left(u_{t}\right)\right) d s-\int_{\Omega}(f \cdot(v-u)-\tau: \varepsilon(v-u)) d x .
\end{aligned}
$$

Here, $\tau$ is any tensor-valued function in $\Sigma(\Omega)$ (it can be considered as an approximation of the true stress $\sigma$ ) and $q \in \widetilde{L}^{2}(\Omega)$ is an approximation of the pressure $p$. In view of the Young-Fenchel inequality, for any $\eta \in L^{2}\left(\Gamma_{2}, \mathbb{R}^{d}\right)$ we have

$$
\int_{\Gamma_{2}}-j\left(u_{t}\right) d s \leqslant \int_{\Gamma_{2}}\left(j^{*}(\eta)-\eta \cdot u_{t}\right) d s,
$$

where $j^{*}$ is the functional conjugate to $j$. In general, $j^{*}$ is defined on a wider set $H^{-1 / 2}$, however, in what follows, we always operate with tensors having summable normal traces on $\Gamma_{2}$ and restrict admissible arguments of $j^{*}$ accordingly. This is motivated by practical requirements because $\eta$ can be considered as an approximation of $\varsigma_{t}$ on $\Gamma_{2}$, which, in numerical applications, is presented by an integrable function.

From (2.2) and (2.3) it follows that

$$
\begin{aligned}
\|\varepsilon(u-v)\|_{\nu}^{2} & \leqslant \int_{\Omega}(\nu \varepsilon(v)-\tau-\mathbb{I} q): \varepsilon(v-u) d x+\int_{\Gamma_{2}}\left(j\left(v_{t}\right)+j^{*}(\eta)-\eta \cdot v_{t}\right) d s \\
& +\int_{\Gamma_{2}} \eta \cdot\left(v_{t}-u_{t}\right) d s-\int_{\Omega}(f \cdot(v-u)-\tau: \varepsilon(v-u)) d x .
\end{aligned}
$$

For any $\eta$ and $\tau$

$$
\mathscr{L}_{\eta, \tau}(w):=\int_{\Omega}(\tau: \varepsilon(w)-f \cdot w) d x+\int_{\Gamma_{2}} \eta \cdot w_{t} d s
$$

is a linear continuous functional on $V_{n, 0}$ the norm of which is defined by the relation

$$
\left\|\mathscr{L}_{\eta, \tau}\right\|:=\sup _{w \in V_{n, 0}} \frac{\left|\mathscr{L}_{\eta, \tau}(w)\right|}{\|\varepsilon(w)\|_{\nu}}
$$


It is easy to see that the set $\operatorname{Ker} \mathscr{L}_{\eta, \tau}$ contains tensor-valued functions that satisfy (in the generalized sense) the equilibrium equation $\operatorname{Div} \tau+f=0$ and the prescribed relation between $\tau$ and $\eta$ on $\Gamma_{2}$ (cf. (1.12)), which states that

$$
\int_{\Gamma_{2}} \varsigma_{t} \cdot w_{t} d s=\int_{\Omega}(\nu \varepsilon(u): \varepsilon(w)-f \cdot w)
$$

for any test function $w \in \mathbb{S}_{n, 0}$. Note that

$$
\begin{aligned}
\int_{\Omega}(\nu \varepsilon(v) & -\tau-\mathbb{I} q): \varepsilon(v-u) d x+\left|\mathscr{L}_{\eta, \tau}(v-u)\right| \\
& \leqslant\|\nu \varepsilon(u)-\tau-\mathbb{I} q\|_{1 / \nu}\|\varepsilon(u-v)\|_{\nu}+\left\|\mathscr{L}_{\eta, \tau}\right\|\|\varepsilon(u-v)\|_{\nu} \\
& \leqslant \frac{\alpha}{2}\|\varepsilon(u-v)\|_{\nu}^{2}+\frac{1}{2 \alpha}\left(\|d(v, q, \tau)\|_{1 / \nu}+\left\|\mathscr{L}_{\eta, \tau}\right\|\right)^{2}
\end{aligned}
$$

where $\alpha$ is a positive number and $d(v, q, \tau):=\nu \varepsilon(v)-\tau-\mathbb{I} q$.

We obtain the first form of the majorant $M_{s l}^{I}$ for the Stokes problem with slip boundary conditions:

$$
\frac{1}{2}\|\varepsilon(u-v)\|_{\nu}^{2} \leqslant M_{s l}^{I}(v, \tau, \eta, q, \alpha):=c_{1}(\alpha) D_{j}\left(v_{t}, \eta\right)+c_{2}(\alpha)\left(\|d(v, q, \tau)\|_{1 / \nu}+\left\|\mathscr{L}_{\eta, \tau}\right\|\right)^{2},
$$

where

$$
D_{j}\left(v_{t}, \eta\right):=\int_{\Gamma_{2}}\left(j\left(v_{t}\right)+j^{*}(\eta)-\eta \cdot v_{t}\right) d s .
$$

The constants $c_{1}$ and $c_{2}$ are defined by the relations

$$
c_{1}(\alpha)=\frac{1}{2-\alpha}, \quad c_{2}(\alpha)=\frac{1}{2 \alpha} c_{1}(\alpha)
$$

where $\alpha \in(0,2)$. This flexibility can be used to balance different parts of the majorants and minimize the values.

By definition,

$$
j^{*}\left(\zeta^{*}\right)=\sup _{\zeta}\left\{\zeta \cdot \zeta^{*}-\varkappa|\zeta|\right\}= \begin{cases}0, & \left|\zeta^{*}\right| \leqslant \varkappa \\ +\infty, & \left|\zeta^{*}\right|>\varkappa .\end{cases}
$$

Hence the majorant is finite provided that $|\eta| \leqslant \varkappa$ on $\Gamma_{2}$. Under this condition,

$$
D_{j}\left(v_{t}, \eta\right)=\int_{\Gamma_{2}}\left(\varkappa\left|v_{t}\right|-\eta \cdot v_{t}\right) d s .
$$

It is not difficult to show that the majorant vanishes if and only if $v, \tau$, and $q$ coincide with $u$, $\sigma$, and $p$ respectively. Indeed, assume that $M_{s l}^{I}(v, \tau, \eta, q, \alpha)=0$. Since the first two terms of the majorant vanish, we see that $\tau=\nu \varepsilon(v)-\mathbb{I} q$ and $\varkappa\left|v_{t}\right|-\eta \cdot v_{t}=0$. Now, from $\left\|\mathscr{L}_{\eta, \tau}\right\|=0$ we conclude that

$$
\int_{\Omega}(\nu \varepsilon(v): \nabla \widetilde{w}-f \cdot \widetilde{w}) d x-(q, \operatorname{div} \widetilde{w})+\int_{\Gamma_{2}} \eta \cdot \widetilde{w}_{t} d s=0 \quad \forall \widetilde{w} \in V_{n, 0} .
$$


We set $\widetilde{w}=v-w$, where $w \in \mathbb{S}_{n, 0}+u_{D}$ and rewrite (2.7) in the form

$$
\int_{\Omega} \nu \varepsilon(v): \varepsilon(v-w) d x+\int_{\Gamma_{2}} \eta \cdot\left(v_{t}-w_{t}\right) d s=(f,(v-w)) .
$$

Since $\eta \cdot v_{t}=j\left(v_{t}\right)$ and

$$
\int_{\Gamma_{2}} \eta \cdot w_{t} d s \leqslant \int_{\Gamma_{2}}\left(j\left(w_{t}\right)+j^{*}(\eta)\right) d s=\int_{\Gamma_{2}} j\left(w_{t}\right) d s,
$$

we conclude that $v$ satisfies (1.14).

So far the estimate is not fully computable because the term $\left\|\mathscr{L}_{\eta, \tau}\right\|$ contains the supremum over an infinite amount of functions (however, majorants of this type may be useful for other purposes, for example, for analysis of errors in Uzawa type iteration methods [20]).

Getting a majorant expressed in terms of computable integrals requires certain reduction of the sets of admissible $\eta$ and $\tau$. Assume that

$$
\tau \in H_{\Gamma_{2}}(\operatorname{Div}, \Omega):=\left\{\tau \in H(\operatorname{Div}, \Omega), \tau_{t} \in L^{2}\left(\Omega, \mathbb{R}^{d}\right) \text { on } \Gamma_{2}\right\},
$$

where $H(\operatorname{Div}, \Omega):=\left\{\tau \in \Sigma(\Omega) \mid \operatorname{Div} \tau \in L^{2}\left(\Omega, \mathbb{R}^{d}\right)\right\}$, and impose an integral type condition

$$
\int_{\Gamma_{2}}\left(\eta+\tau_{t}\right) d s=0
$$

We integrate by parts, recall that $w \in \mathbb{S}_{n, 0}$, and represent $\mathscr{L}_{\eta, \tau}$ in the form

$$
\mathscr{L}_{\eta, \tau}(w)=-\int_{\Omega}(f+\operatorname{Div} \tau) \cdot w d x+\int_{\Gamma_{2}}\left(\eta+\tau_{t}\right) \cdot w_{t} d s .
$$

The first integral is easily estimated by (1.8) (valid for vector-valued functions vanishing on $\Gamma_{1}$ )

$$
\int_{\Omega}(f+\operatorname{Div} \tau) \cdot w d x \leqslant C_{\Omega}\|f+\operatorname{Div} \tau\|\|\varepsilon(w)\| .
$$

Certainly, in this case, we need to know $C_{\Omega}$ or have a realistic upper bound of it. In many cases, such a bound can be indeed found (cf., for example, a discussion related to this question and practical recommendations in [33]). In other cases, we suggest a different way based on the idea of domain decomposition (in the context of a posteriori estimates it is systematically considered in [15]). This method is briefly presented in Remark 2.1 below.

The last integral is estimated by means of the Poincaré type inequality

$$
\|\varphi\|_{\Gamma_{2}} \leqslant \widetilde{C}_{\Gamma_{2}}(\Omega)\|\nabla \varphi\|
$$

which holds for any $\varphi \in H^{1}(\Omega)$ such that $\{\varphi\}_{\Gamma_{2}}=0$. For such domains as parallelepipeds, triangles, and tetrahedrons, the constant $\widetilde{C}_{\Gamma_{2}}$ has been found analytically or numerically (cf. $[36,37]$ and the references therein).

It is worth noting that the constants $\widetilde{C}_{\Gamma_{2}}(\Omega)$ possess a certain monotonicity property, namely, if $\Omega^{\prime} \subset \Omega$ and both domains have the same boundary part $\Gamma_{2}$ (where the functions have traces 
with zero mean), then $\widetilde{C}_{\Gamma_{2}}(\Omega) \leqslant \widetilde{C}_{\Gamma_{2}}\left(\Omega^{\prime}\right)$. Hence, if $\widetilde{C}_{\Gamma_{2}}\left(\Omega^{\prime}\right)$ is known for a "simple" domain $\Omega^{\prime}$, then this constant is valid for the whole $\Omega$. This fact allows us to obtain bounds of the constant for a wide spectrum of domains. For example, let the "simple" domain be a right triangle with both legs having the same length (cf. Figure). We have three possible options: $\Gamma_{2}$ coincides with (a) one leg, (b) the union of two legs, and (c) the hypotenuse. In all these cases, the constants $\widetilde{C}_{\Gamma_{2}}(\Omega)$ were found analytically. For example, if $\Gamma$ is a leg (of length $h$ ), then

$$
\widetilde{C}_{\Gamma_{2}}(\Omega) \leqslant \sqrt{h}(\zeta \tanh \zeta)^{-1 / 2},
$$

where $\zeta \approx 2.3650$ is the root of the equation $\tan (z)+\tanh (z)=0$ in the interval $(0, \pi)$. If $\Gamma$ coincides with the hypotenuse of the right triangle with legs of the size $h$, then

$$
\widetilde{C}_{\Gamma_{2}}(\Omega) \leqslant \sqrt{h} .
$$

Using these results and applying affine mappings, one can deduce the constants for any other nondegenerate triangle (cf. [36]). Therefore, finding the constants is not a difficult task (at least in the case where $\Gamma_{2}$ is a boundary of a polygonal domain).

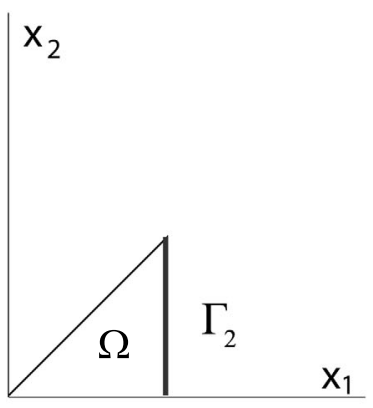

(a)

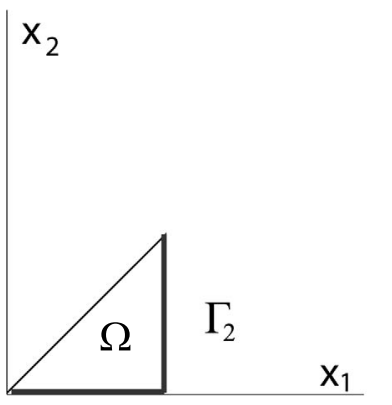

(b)

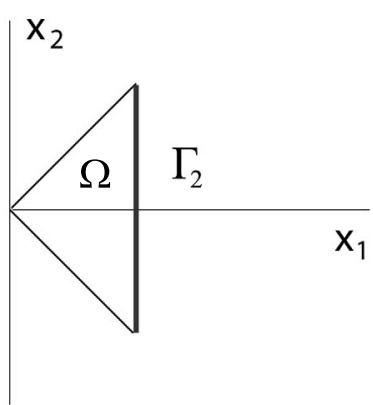

(c)

Figure. Right triangles: (a) $\Gamma_{2}$ is a leg, $\Gamma_{2}$ is the union of two legs, (c) $\Gamma_{2}$ is the hypotenuse.

It is clear that (2.10) also holds for vector-valued functions. Moreover, since the rigid motions are excluded by the boundary condition on $\Gamma_{1}$, we can rewrite it in terms of the symmetric gradient tensor, i.e.,

$$
\|w\|_{\Gamma_{2}}=\widehat{C}_{\Gamma_{2}}(\Omega)\|\varepsilon(w)\|
$$

provided that $w \in V$ satisfies the conditions $\left\{w_{i}\right\}_{\Gamma_{2}}=0$ for $i \in 1, \ldots, d$ (in this case, the constant $\widehat{C}_{\Gamma_{2}}(\Omega)$ can be obtained from the constant in (2.10) if we multiply it by the Korn constant).

In view of (2.11) and (2.8), we have

$$
\begin{aligned}
\int_{\Gamma_{2}}\left(\eta+\tau_{t}\right) \cdot w_{t} d s & =\int_{\Gamma_{2}}\left(\eta+\tau_{t}\right) \cdot\left(w_{t}-\left\{w_{t}\right\}_{\Gamma_{2}}\right) d s \\
& \leqslant\left\|\eta+\tau_{t}\right\|_{\Gamma_{2}}\left\|w-\{w\}_{\Gamma_{2}}\right\| \leqslant \widehat{C}_{\Gamma_{2}}\left\|\eta+\tau_{t}\right\|_{\Gamma_{2}}\|\varepsilon(w)\|
\end{aligned}
$$

and

$$
\left\|\mathscr{L}_{\eta, \tau}\right\| \leqslant C_{\Omega}\|f+\operatorname{Div} \tau\|+\widehat{C}_{\Gamma_{2}}\left\|\eta+\tau_{t}\right\|_{\Gamma_{2}} .
$$

We recall (2.5) and arrive at the following result. 
Theorem 2.1. For any $v \in \mathbb{S}_{n, 0}+u_{D}, \tau \in H_{\Gamma_{2}}(\operatorname{Div}, \Omega), \eta \in L^{2}\left(\Gamma_{2}, \mathbb{R}^{d}\right)(|\eta| \leqslant \varkappa), q \in$ $\widetilde{L}^{2}(\Omega)$, and $\alpha \in(0,2)$ the error is subject to the estimate

$$
\frac{1}{2}\|\varepsilon(u-v)\|_{\nu}^{2} \leqslant M_{s l}^{I I}(v, \tau, \eta, q, \alpha),
$$

where the majorant is defined by the relation

$$
\begin{aligned}
M_{s l}^{I I}(v, \tau, \eta, q, \alpha) & :=c_{1}(\alpha) D_{j}\left(v_{t}, \eta\right) \\
& +c_{2}(\alpha)\left(\|d(v, q, \tau)\|_{1 / \nu}+C_{\Omega}\|f+\operatorname{Div} \tau\|+\widehat{C}_{\Gamma_{2}}\left\|\eta+\tau_{t}\right\|_{\Gamma_{2}}\right)^{2}
\end{aligned}
$$

and the constants are defined in (2.6), (2.9), and (2.10).

Note that the majorant in (2.12) is expressed in terms of integrals (unlike the majorant $\left.M_{s l}^{I}(v, \tau, \eta, q, \alpha)\right)$ and contains only known functions $(v, \tau$, and $\eta$ are approximations of different components of the exact solution). As above, $\alpha$ is any number in $(0,2)$. It can be used to properly balance parts of the majorant.

If $M_{s l}^{I I}(v, \tau, \eta, q, \alpha)=0$, then $\left\|\mathscr{L}_{\eta, \tau}\right\|=0$, and, repeating the same arguments as above, we conclude that $v$ coincides with the exact solution.

Remark 2.1. We can deduce a different form of the majorant if (2.9) is replaced by the estimate

$$
\int_{\Omega}(f+\operatorname{Div} \tau) \cdot w d x \leqslant \sum_{i=1}^{N} C_{R D}\left(\Omega_{i}\right)\|f+\operatorname{Div} \tau\|_{\Omega_{i}}\|\varepsilon(w)\|_{\Omega_{i}}
$$

which holds provided that

$$
\int_{\Omega_{i}}(f+\operatorname{Div} \tau) \cdot \eta d x=0 \quad \forall \eta \in \mathscr{E}_{0}:=\left\{\varepsilon(\eta) \equiv 0 \text { in } \Omega_{i}\right\}
$$

for any Lipschitz subdomain $\Omega_{i}, i=1,2, \ldots, N$, such that $\Omega_{i} \cap \Omega_{j}=\emptyset$ if $i \neq j$ and $\bar{\Omega}=\cup_{i=1}^{N} \bar{\Omega}_{i}$ (cf. [15, Chapter 7]). Here, $C_{R D}\left(\Omega_{i}\right)$ are constants in the inequalities $\|v\|_{\Omega_{i}} \leqslant C_{R D}\left(\Omega_{i}\right)\|\varepsilon(v)\|_{\Omega_{i}}$ which hold for functions orthogonal to the kernel of the operator $\varepsilon$ (i.e., they are orthogonal to rigid deflections). The existence of $C_{R D}\left(\Omega_{i}\right)$ is easily proved by standard compactness arguments. Then we obtain

$$
\frac{1}{2}\|\varepsilon(u-v)\|_{\nu}^{2} \leqslant \widetilde{M}_{s l}^{I I}(v, \tau, \eta, q, \alpha)
$$

where

$$
\begin{aligned}
\widetilde{M}_{s l}^{I I}(v, \tau, \eta, q, \alpha) & :=c_{1}(\alpha) D_{j}\left(v_{t}, \eta\right) \\
& +c_{2}(\alpha)\left(\|d(v, q, \tau)\|_{1 / \nu}+\sqrt{\sum_{i=1}^{N} C_{R D}^{2}\left(\Omega_{i}\right)\|f+\operatorname{Div} \tau\|_{\Omega_{i}}^{2}}+\widehat{C}_{\Gamma_{2}}\left\|\eta+\tau_{t}\right\|_{\Gamma_{2}}\right)^{2} .
\end{aligned}
$$

The estimates (2.12) and (2.15) are valid only for divergence free functions. This fact may lead to certain difficulties in practical applications because approximations of the velocity field obtained by numerical methods often satisfy this condition only approximately. Therefore, our next goal is to extend the estimates to a wider class of functions which may not exactly satisfy 
the divergence free condition (1.2). This can be done by using the same ideas as has been earlier used in the case of the Stokes and Oseen problems [16, 15].

2.2. Estimates for approximations in $V_{n, 0}+u_{D}$. Assume that $v \in V_{n, 0}+u_{D}$. For any $v_{0} \in \mathbb{S}_{0}$ and $\beta>0$

$$
\begin{aligned}
\|\varepsilon(u-v)\|_{\nu}^{2} & \leqslant\left(\left\|\nabla\left(u-v_{0}\right)\right\|_{\nu}+\| \nabla\left(v-v_{0} \|_{\nu}\right)^{2}\right. \\
& \leqslant(1+\beta)\left\|\nabla\left(u-v_{0}\right)\right\|_{\nu}^{2}+\left(1+\frac{1}{\beta}\right)\left\|\nabla\left(v-v_{0}\right)\right\|_{\nu}^{2} .
\end{aligned}
$$

The first term on the right-hand side of (2.16) is estimated from above by $M_{s l}^{I I}\left(v_{0}, \tau, \eta, q, \alpha\right)$ (or by $\left.\widetilde{M}_{s l}^{I I}\left(v_{0}, \tau, \eta, q, \alpha\right)\right)$.

We consider the respective parts of the majorant. First, we note that

$$
\begin{aligned}
& \left\|d\left(v_{0}, q, \tau\right)\right\|_{1 / \nu} \leqslant\|\nu \varepsilon(v)-\tau-\mathbb{I} q\|_{1 / \nu}+\left\|\nu \nabla\left(v-v_{0}\right)\right\|_{1 / \nu} \\
& \leqslant\|\nu \varepsilon(v)-\tau-\mathbb{I} q\|_{1 / \nu}+\left\|\nabla\left(v-v_{0}\right)\right\|_{\nu} \leqslant\|d(v, q, \tau)\|_{1 / \nu}+\sqrt{\bar{\nu}}\left\|\nabla\left(v-v_{0}\right)\right\| .
\end{aligned}
$$

Next,

$$
\begin{aligned}
& \int_{\Gamma_{2}}\left(j\left(v_{0 t}\right)+j^{*}(\eta)-\eta \cdot v_{0 t}\right) d s=D_{j}\left(v_{t}, \eta\right)+\int_{\Gamma_{2}}\left(\eta \cdot v_{t}-j\left(v_{t}\right)\right) d s \\
& \leqslant D_{j}\left(v_{t}, \eta\right)+\int_{\Gamma_{2}}\left(\left(\eta+\tau_{t}\right) \cdot v_{t}+j^{*}\left(-\tau_{t}\right)\right) d s \leqslant D_{j}\left(v_{t}, \eta\right)+\int_{\Gamma_{2}}\left(\eta+\tau_{t}\right) \cdot v_{t} d s
\end{aligned}
$$

provided that $\left|\tau_{t}\right| \leqslant \varkappa$.

We recall $(2.8)$ and that $v_{0}$ vanishes on the boundary $\Gamma_{1}$. Then

$$
\begin{aligned}
\int_{\Gamma_{2}}\left(\eta+\tau_{t}\right) \cdot v_{t} d s & =\int_{\Gamma_{2}}\left(\eta+\tau_{t}\right) \cdot\left(v_{t}-v_{0 t}\right) d s \\
& \leqslant \widehat{C}_{\Gamma}\left\|\eta+\tau_{t}\right\|\left\|\nabla\left(v_{t}-v_{0 t}\right)\right\| \leqslant \widehat{C}_{\Gamma}\left\|\eta+\tau_{t}\right\|\left\|\nabla\left(v-v_{0}\right)\right\| .
\end{aligned}
$$

Hence

$$
\begin{aligned}
M_{s l}^{I I}\left(v_{0}, \tau, \eta, q, \alpha, \beta\right) & \leqslant c_{1}(\alpha) D_{j}\left(v_{t}, \eta\right)+c_{1}(\alpha) \widehat{C}_{\Gamma}\left\|\eta+\tau_{t}\right\|\left\|\nabla\left(v-v_{0}\right)\right\| \\
& +c_{2}(\alpha)\left(R(v, q, \tau, \eta)+\sqrt{\bar{\nu}}\left\|\nabla\left(v-v_{0}\right)\right\|\right)^{2}
\end{aligned}
$$

where

$$
R(v, q, \tau, \eta):=\|d(v, q, \tau)\|_{1 / \nu}+\left(C_{\Omega}\|f+\operatorname{Div} \tau\|+\widehat{C}_{\Gamma}\left\|\eta+\tau_{t}\right\|_{\Gamma_{2}}\right) .
$$

Now, we recall that the distance between a vector-valued function $v \in H^{1}\left(\Omega, \mathbb{R}^{d}\right)$ satisfying the homogeneous boundary conditions on $\Gamma_{1}$ and $\mathbb{S}_{0}$ is subject to the estimate (cf., for example, $[16])$

$$
\inf _{v_{0} \in \mathbb{S}_{0}}\left\|\nabla\left(v-v_{0}\right)\right\| \leqslant \mathbb{C}_{\Omega}\|\operatorname{div} v\|
$$

In view of (2.19), the respective terms in (2.16), (2.17), and (2.18) are estimated by the norm of $\operatorname{div} v$. This result can be extended to the case, where the functions vanish only on a part 
$\Gamma_{1}$ of $\Gamma$ provided that $v$ additionally satisfies $\{\operatorname{div} v\}_{\Omega}=0$ (cf. [15, Chapter 6, Section 2.6]). It is worth noting that the distance estimate (2.19) is important not only for incompressible viscous problems. In [38], it was used to derive a new (fully computable) bound of the limit load parameter in the Hencky plasticity problems.

Thus, we arrive at the following result.

Theorem 2.2. For any $\alpha \in(0,2), \beta>0,\left|\tau_{t}\right| \leqslant \varkappa$, and $|\eta| \leqslant \varkappa$ the distance between $u$ and $v$ is subject to the estimate

$$
\|\varepsilon(u-v)\|_{\nu}^{2} \leqslant M_{s l}^{I I I}(v, \tau, \eta, q, \alpha, \beta)
$$

where

$$
\begin{gathered}
M_{s l}^{I I I}(v, \tau, \eta, q, \alpha, \beta):=(1+\beta)\left(c_{1}(\alpha) D_{j}\left(v_{t}, \eta\right)+c_{2}(\alpha) R^{2}(v, q, \tau, \eta)\right. \\
\left.+H(v, q, \tau, \eta, \alpha) \mathbb{C}_{\Omega}\|\operatorname{div} v\|+c_{3}(\alpha, \beta) \mathbb{C}_{\Omega}^{2}\|\operatorname{div} v\|^{2}\right), \\
c_{3}(\alpha, \beta)=\left(c_{2}(\alpha)+\frac{1}{2 \beta}\right) \bar{\nu}, \text { and } \\
H(v, q, \tau, \eta, \alpha):=\widehat{C}_{\Gamma}\left\|\eta+\tau_{t}\right\|_{\Gamma_{2}}+2 c_{2}(\alpha) \sqrt{\bar{\nu}} R(v, q, \tau, \eta) .
\end{gathered}
$$

Remark 2.2. All the terms of the majorant are nonnegative. If $M_{s l}^{I I I}(v, \tau, \eta, q, \alpha, \beta)=0$, then $\operatorname{div} v=0$, and, by the same arguments as for $M_{s l}^{I I}$, we conclude that the functions entering the majorant coincide with the exact velocity, pressure, and stress. By the same arguments as above, the constant $C_{\Omega}$ entering $R(v, q, \tau, \eta)$ can be replaced by a collection of local constants associated with subdomains $\Omega_{i}$.

Further modifications of the majorant are related to the condition (2.19). In [17], it was shown that the distance between $v$ and $\mathbb{S}_{0}$ has another upper bound based on local constants $\mathbb{C}_{i}$ associated with $\Omega_{i}$.

Lemma 2.1. Let $v \in V$ satisfy

$$
\{\operatorname{div} v\}_{\Omega_{i}}=0, \quad i=1,2, \ldots, N .
$$

Then,

$$
\inf _{v_{0} \in \mathbb{S}_{0}(\Omega)}\left\|\nabla\left(v-v_{0}\right)\right\|^{2} \leqslant d_{N}^{2}(v):=\sum_{i=1}^{N} \mathbb{C}_{\Omega_{i}}^{2}\|\operatorname{div} v\|_{\Omega_{i}}^{2} .
$$

By Lemma 2.1, we modify the estimate (2.20) as follows

$$
\|\varepsilon(u-v)\|_{\nu}^{2} \leqslant \widetilde{M}_{s l}^{I I I}(v, \tau, \eta, q, \alpha, \beta),
$$

where

$$
\begin{aligned}
\widetilde{M}_{s l}^{I I I}(v, \tau, \eta, q, \alpha, \beta) & :=(1+\beta)\left(c_{1}(\alpha) D_{j}\left(v_{t}, \eta\right)+c_{2}(\alpha) R^{2}(v, q, \tau, \eta)\right. \\
& \left.+H(v, q, \tau, \eta, \alpha) d_{N}(v)+c_{3}(\alpha, \beta) d_{N}^{2}(v)\right)
\end{aligned}
$$


which uses the constants $\mathbb{C}_{\Omega_{i}}$ in the LBB inequality for local subdomains.

It should be remarked that the constant $\mathbb{C}_{\Omega}$ may be unknown (if $\Omega$ is a complicated domain). However, $\Omega$ may admit a decomposition into simpler domains for which the corresponding constants (or upper bounds of them) may be known. At least, for $2 \mathrm{D}$ star-shaped domains with respect to a ball such estimates are known [27]. Therefore, the estimate (2.23) is practically more convenient than (2.20). Certainly, in this estimate, $v$ must satisfy the additional conditions (2.21). However, these integral type relations do not produce essential technical difficulties because any reasonable approximation of $u \in \mathbb{S}(\Omega)$ should satisfy the incompressibility condition in $\Omega_{i}$, at least, in the integral (mean) sense. If, by some reasons, they do not satisfy it, then they should be corrected by a post-processing procedure changing normal components of the velocity on $\partial \Omega_{i}$. Typically, the number $N$ (amount of subdomains $\Omega_{i}$ ) is not large, so that such type "correction" procedures could be quite cheap.

\section{Leak Boundary Condition}

Analysis of problems with the leak boundary conditions follows the same lines as for the slip ones. Below we expose the results supplied with brief comments.

3.1. Estimates for functions in $\mathbb{S}_{t, 0}+u_{D}$. From (1.15) it follows that

$$
a(u-v, v-u)+\int_{\Gamma_{2}}\left(j\left(v_{n}\right)-j\left(u_{n}\right)\right) d s \geqslant(f, v-u)-a(v, v-u),
$$

where $v \in \mathbb{S}_{t, 0}+u_{D}$ is an approximation of $u$.

Let $\tau \in \Sigma(\Omega)$, and let $q \in \widetilde{L}^{2}(\Omega)$. By (3.1), we obtain

$$
\begin{aligned}
\|\varepsilon(u-v)\|_{\nu}^{2} \leqslant & \int_{\Omega}(\nu \varepsilon(v)-\tau-\mathbb{I} q): \varepsilon(v-u) d x \\
& +\int_{\Gamma_{2}}\left(j\left(v_{n}\right)-j\left(u_{n}\right)\right) d s-\int_{\Omega}(f \cdot(v-u)-\tau: \varepsilon(v-u)) d x \\
\leqslant & \int_{\Omega}(\nu \varepsilon(v)-\tau-\mathbb{I} q): \varepsilon(v-u) d x+\int_{\Gamma_{2}}\left(j\left(v_{n}\right)+j^{*}(\eta)-\eta v_{n}\right) d s \\
& +\int_{\Gamma_{2}} \eta\left(v_{n}-u_{n}\right) d s-\int_{\Omega}(f \cdot(v-u)-\tau: \varepsilon(v-u)) d x .
\end{aligned}
$$

Define the functional

$$
\mathscr{L}_{\eta, \tau}(w):=\int_{\Omega}(\tau: \varepsilon(w)-f \cdot w) d x+\int_{\Gamma_{2}} \eta w_{n} d s
$$

and the corresponding norm

$$
\left\|\mathscr{L}_{\eta, \tau}\right\|:=\sup _{w \in V_{t, 0}} \frac{\left|\mathscr{L}_{\eta, \tau}(w)\right|}{\|\varepsilon(w)\|_{\nu}} .
$$


The set $\operatorname{Ker} \mathscr{L}_{\eta, \tau}$ contains tensor-valued functions that satisfy (in a generalised sense) the equilibrium equation Div $\tau+f$ and the relation between $\tau$ and $\eta$ on $\Gamma_{2}$. We reform the right-hand side of (3.2) and, by the same arguments as for the slip boundary condition, deduce the estimate

$$
\frac{1}{2}\|\varepsilon(u-v)\|_{\nu}^{2} \leqslant M_{l e}^{I}(v, \tau, \eta, q, \alpha)
$$

where $M_{l e}^{I}(v, \tau, \eta, q, \alpha)=: c_{1}(\alpha) D_{j}\left(v_{n}, \eta\right)+c_{2}(\alpha)\left(\|d(v, q, \tau)\|_{1 / \nu}+\left\|\mathscr{L}_{\eta, \tau}\right\|\right)^{2}$ and

$$
D_{j}\left(v_{n}, \eta\right):=\int_{\Gamma_{2}}\left(j\left(v_{n}\right)+j^{*}(\eta)-\eta v_{n}\right) d s .
$$

The coefficients $c_{i}(\alpha), i=1,2$, and $d(v, q, \tau)$ are defined as in the previous section, and $\alpha \in(0,2)$. If the function $v$ satisfies the condition $\left|v_{n}\right| \leqslant \varkappa$ (what is a natural requirement for a reasonable approximation of $u$ ), then the majorant is finite and

$$
D_{j}\left(v_{n}, \eta\right)=\int_{\Gamma_{2}}\left(\varkappa\left|v_{n}\right|-\eta v_{n}\right) d s .
$$

Assume that $M_{l e}^{I}(v, \tau, \eta, q, \alpha)=0$. Then $\tau=\nu \varepsilon(v)-\mathbb{I} q$ and $\varkappa\left|v_{n}\right|-\eta v_{n}=0$. Also, $\left\|\mathscr{L}_{\eta, \tau}\right\|=0$. Therefore, for any $w \in \mathbb{S}_{t, 0}$ we have

$$
\int_{\Omega}(\nu \varepsilon(v): \varepsilon(w)-f \cdot w) d x-(q, \operatorname{div} w)+\int_{\Gamma_{2}} \eta w_{n} d s=0 .
$$

We can set the test function in the form $v-w$, where $w \in S_{t, 0}+u_{D}$, and rewrite (3.4) in the form

$$
\int_{\Omega} \nu \varepsilon(v): \varepsilon(v-w) d x+\int_{\Gamma_{2}} \eta\left(v_{n}-w_{n}\right) d s=\int_{\Omega} f \cdot(v-w) d x .
$$

Note that $\eta v_{n}=j\left(v_{n}\right)$ and

$$
\int_{\Gamma_{2}} \eta w_{n} d s \leqslant \int_{\Gamma_{2}} j\left(w_{n}\right) d s
$$

We conclude that $v$ satisfies

$$
\int_{\Omega} \nu \varepsilon(v): \varepsilon(v-w) d x+\int_{\Omega} f \cdot(w-v) d x \leqslant \int_{\Gamma_{2}} j\left(w_{n}\right) d s-\int_{\Gamma_{2}} j\left(v_{n}\right) d s
$$

for any $w$ and, therefore, $v$ satisfies (1.15).

The norm $\left\|\mathscr{L}_{\eta, \tau}\right\|$ is estimated from above by the same arguments as in Section 2. We impose additional conditions on admissible $\eta$ and $\tau$, namely,

$$
\tau \in H_{\Gamma_{2}}(\operatorname{Div}, \Omega):=\left\{\tau \in H(\operatorname{Div}, \Omega), \tau_{n n} \in L^{2}\left(\Omega, \mathbb{R}^{d}\right) \text { on } \Gamma_{2}\right\}
$$

and

$$
\int_{\Gamma_{2}}\left(\eta+\tau_{n n}\right) d s=0 .
$$


It is clear that for $\tau=\sigma$ and $\eta=\sigma_{n}$ this condition holds. Moreover, for any pair of approximations $(\eta$ and $\tau$ ) satisfaction of this integral condition does not lead to essential difficulties. With these conditions, we rewrite $\mathscr{L}_{\eta, \tau}(w)$ as follows:

$$
\mathscr{L}_{\eta, \tau}(w)=-\int_{\Omega}(f+\operatorname{Div} \tau \cdot w) d x+\int_{\Gamma_{2}}\left(\eta+\tau_{n n}\right) w_{n} d s .
$$

Since

$$
\begin{aligned}
& \int_{\Gamma_{2}}\left(\eta+\tau_{n n}\right) \cdot w_{n} d s \leqslant \widehat{C}_{\Gamma}\left\|\eta+\tau_{n n}\right\|_{\Gamma_{2}}\|\varepsilon(w)\|, \\
& \int_{\Omega}(f+\operatorname{Div} \tau \cdot w) d x \leqslant C_{\Omega}\|f+\operatorname{Div} \tau\|\|\varepsilon(w)\|,
\end{aligned}
$$

we find that

$$
\left\|\mathscr{L}_{\eta, \tau}\right\| \leqslant C_{\Omega}\|f+\operatorname{Div} \tau\|+\widehat{C}_{\Gamma}\|\eta-\tau \cdot n\|_{\Gamma_{2}} .
$$

Hence we arrive at the result similar to Theorem 2.1.

Theorem 3.1. For any $v \in \mathbb{S}_{t, 0}+u_{D}, \tau \in H_{\Gamma_{2}}(\operatorname{Div}, \Omega), \eta \in L^{2}\left(\Gamma_{2}\right)(|\eta| \leqslant \varkappa), q \in \widetilde{L}^{2}(\Omega)$, and $\alpha \in(0,2)$, the error is subject to the estimate

$$
\frac{1}{2}\|\varepsilon(u-v)\|_{\nu}^{2} \leqslant M_{l e}^{I I}(v, \tau, \eta, q, \alpha)
$$

where

$$
\begin{aligned}
M_{l e}^{I I}(v, \tau, \eta, q, \alpha) & :=c_{1}(\alpha) D_{j}\left(v_{n}, \eta\right) \\
& +c_{2}(\alpha)\left(\|d(v, q, \tau)\|_{1 / \nu}+C_{\Omega}\|f+\operatorname{Div} \tau\|+\widehat{C}_{\Gamma}\left\|\eta+\tau_{n n}\right\|_{\Gamma_{2}}\right)^{2} .
\end{aligned}
$$

The estimate (3.5) has the same meaning and properties as the estimate (2.12).

If $M_{l e}^{I I}(v, \tau, \eta, q, \alpha)=0$, then $d(v, q, \tau)=0, D_{j}\left(v_{n}, \eta\right)=0,\left\|\mathscr{L}_{\eta, \tau}\right\|=0$, and we conclude that $v, \tau$, and $q$ coincide with $u, \sigma$, and $p$ respectively.

If $\tau$ satisfies the mean value conditions (2.14) in subdomains $\Omega_{i}$, then the estimates $(2.13)$ and (3.5) imply a somewhat different form of majorant based upon different constants (which is analogous to $(2.15))$ :

$$
\begin{aligned}
\widetilde{M}_{l e}^{I I}(v, \tau, \eta, q, \alpha) & :=c_{1}(\alpha) D_{j}\left(v_{n}, \eta\right) \\
& +c_{2}(\alpha)\left(\|d(v, q, \tau)\|_{1 / \nu}+\sqrt{\sum_{i=1}^{N} C_{R D}^{2}\left(\Omega_{i}\right)\|f+\operatorname{Div} \tau\|_{\Omega_{i}}^{2}}+\widehat{C}_{\Gamma}\left\|\eta+\tau_{n n}\right\|_{\Gamma_{2}}\right)^{2}
\end{aligned}
$$

All comments in Remark 2.1 remain true for $\widetilde{M}_{l e}^{I I}(v, \tau, \eta, q, \alpha)$.

3.2. Estimates for approximations in $V_{t, 0}+u_{D}$. An extension of the estimate to $v \in V_{t, 0}+u_{D}$ follows from (2.16), (2.17), and the relation

$$
\int_{\Gamma_{2}}\left(j\left(v_{0 n}\right)+j^{*}(\eta)-\eta \cdot v_{0 n}\right) d s=D_{j}\left(v_{t}, \eta\right)+\int_{\Gamma_{2}}\left(\eta \cdot v_{n}-j\left(v_{n}\right)\right) d s
$$




$$
\leqslant D_{j}\left(v_{n}, \eta\right)+\int_{\Gamma_{2}}\left(\eta+\tau_{n n}\right) \cdot v_{n} d s,
$$

where $v_{0} \in \mathbb{S}_{0}$ and $\left|\tau_{n n}\right| \leqslant \varkappa$. By $(2.8)$, we obtain

$$
\begin{aligned}
\int_{\Gamma_{2}}\left(\eta+\tau_{n n}\right) \cdot v_{n} d s & =\int_{\Gamma_{2}}\left(\eta+\tau_{n n}\right) \cdot\left(v_{n}-v_{0 n}\right) d s \\
& \leqslant \widehat{C}_{\Gamma}\left\|\eta+\tau_{n n}\right\|\left\|\nabla\left(v_{n}-v_{0 n}\right)\right\| \leqslant \widehat{C}_{\Gamma}\left\|\eta+\tau_{n n}\right\|\left\|\nabla\left(v-v_{0}\right)\right\| .
\end{aligned}
$$

Therefore,

$$
\begin{aligned}
M_{l e}^{I I}\left(v_{0}, \tau, \eta, q, \alpha, \beta\right) & \leqslant c_{1}(\alpha) D_{j}\left(v_{n}, \eta\right)+c_{1}(\alpha) \widehat{C}_{\Gamma}\left\|\eta+\tau_{n n}\right\|\left\|\nabla\left(v-v_{0}\right)\right\| \\
& +c_{2}(\alpha)\left(R(v, q, \tau, \eta)+\sqrt{\bar{\nu}}\left\|\nabla\left(v-v_{0}\right)\right\|\right)^{2}
\end{aligned}
$$

where

$$
R(v, q, \tau, \eta):=\|d(v, q, \tau)\|_{1 / \nu}+\left(C_{\Omega}\|f+\operatorname{Div} \tau\|+\widehat{C}_{\Gamma}\left\|\eta+\tau_{n n}\right\|_{\Gamma_{2}}\right) .
$$

Now, we recall (2.19) and arrive at the following result.

Theorem 3.2. For any $\alpha \in(0,2), \beta>0,\left|\tau_{n n}\right| \leqslant \varkappa$, and $|\eta| \leqslant \varkappa$

$$
\|\varepsilon(u-v)\|_{\nu}^{2} \leqslant M_{l e}^{I I I}(v, \tau, \eta, q, \alpha, \beta)
$$

where

$$
\begin{aligned}
& M_{l e}^{I I I}(v, \tau, \eta, q, \alpha, \beta):=(1+\beta)\left(c_{1}(\alpha) D_{j}\left(v_{n}, \eta\right)+c_{2}(\alpha) R^{2}(v, q, \tau, \eta)\right. \\
&\left.+H(v, q, \tau, \eta, \alpha) \mathbb{C}_{\Omega}\|\operatorname{div} v\|+c_{3}(\alpha, \beta) \mathbb{C}_{\Omega}^{2}\|\operatorname{div} v\|^{2}\right), \\
& H(v, q, \tau, \eta, \alpha):=\widehat{C}_{\Gamma}\left\|\eta+\tau_{n n}\right\|+2 c_{2}(\alpha) \sqrt{\bar{\nu}} R(v, q, \tau, \eta) .
\end{aligned}
$$

Remark 3.1. The majorant $M_{l e}^{I I I}(v, \tau, \eta, q, \alpha, \beta)$ in (3.6) is quite analogous to the majorant $M_{s l}^{I I I}(v, \tau, \eta, q, \alpha, \beta)$ in (2.20). It consists of nonnegative terms and vanishes if and only if $v$, $\tau$, and $q$ coincide with the respective exact solutions. By the same arguments as above, the constant $C_{\Omega}$ entering $R(v, q, \tau, \eta)$ can be replaced by a collection of local constants associated with subdomains $\Omega_{i}$. Also, we can use $(2.22)$ and replace the term containing $\mathbb{C}_{\Omega}\|\operatorname{div} v\|$ by the quantity $d_{N}(v)$ (cf. $(2.21)$ ) containing the constants $\mathbb{C}_{\Omega_{i}}$.

\section{References}

1. H. Fujita, "A mathematical analysis of motions of viscous incompressible fluid under leak or slip boundary conditions," RIMS Kokyuroku, 888, 199-216 (1994).

2. H. Fujita, "Non-stationary Stokes flows under leak boundary conditions of friction type," J. Comput. Math. 19, No. 1, 1-8 (2001). 
3. H. Fujita, "A coherent analysis of Stokes flows under boundary conditions of friction type," J. Comput. Appl. Math. 149, 57-69 (2002).

4. H. Kawarada, H. Fujita, and H. Suito, "Wave breaking phenomenon on the shore and coastal ecosystems," GAKUTO Int. Ser., Math. Sci. Appl. 12, 65-74 (1999)

5. H. Kawarada and H. Suito, "Numerical simulation of spilled oil drifted on the sloping beach," In: Computational Fluid and Solid Mechanics, pp. 876-878, Elsevier, Amsterdam etc. (2001).

6. M. Feistauer, Mathematical Methods in Fluid Dynamics, Wiley, New York (1993).

7. V. Girault and P. A. Raviart, Finite Element Approximation of the Navier-Stokes Equations, Springer, Berlin etc. (1979).

8. M. Fortin and R. Glowinski, Augmented Lagrangian Methods: Applications to the Numerical Solution of Boundary-Value Problems, North-Holland, Amsterdam etc. (1983).

9. R. Glowinski, Numerical Methods for Nonlinear Variational Problems, Springer, New York etc. (1984).

10. J. Haslinger and P. D. Panagiotopoulos, "The reciprocal variational approach to the Signorini problem with friction. Approximation results," Proc. R. Soc. Edinb., Sect. A 98, No. 3-4, 365-383 (1984).

11. J. Haslinger, I. Hlaváček, and J. Nečas, Numerical methods for unilateral problems in solid mechanics, In: Handbook of Numerical Analysis, Vol. 4, pp. 313-485, Elsevier (1996).

12. J. Haslinger and J. Stebel, "Stokes problem with a solution dependent slip bound: Stability of solutions with respect to domains," ZAMM, Z. Angew. Math. Mech. 96, No. 9, 10491060, (2016).

13. O. Pironneau, Finite Element Methods for Fluids, John Wiley \& Sons, Chichester etc. (1989).

14. R. Temam, Navier-Stokes Equations. Theory and Numerical Analysis, North-Holland, Amsterdam etc. (1977).

15. S. Repin, A Posteriori Estimates for Partial Differential Equations, de Gruyter, Berlin (2008).

16. S. Repin, "A posteriori estimates for the Stokes problem," J. Math. Sci., New York 109, No. 5, 1950-1964 (2002).

17. S. Repin, "Estimates of the distance to the set of solenoidal vector fields and applications to a posteriori error control," Comput. Methods Appl. Math. 15, No. 4. 515-530 (2015).

18. S. Repin and J. Valdman, "Functional a posteriori error estimates for problems with nonlinear boundary conditions," J. Numer. Math. 16, No. 1, 51-81 (2008).

19. P. Neittaanmäki, S. Repin, and J. Valdman, "Estimates of deviations from exact solutions for elasticity problems with nonlinear boundary conditions," Russ. J. Numer. Anal. Math. Model. 28, No. 6, 597-630 (2013).

20. M. Nokka and S. Repin, "A posteriori error bounds for approximations of the Oseen problem and applications to Uzawa iteration algorithm," Comput. Methods Appl. Math. 14, No. 3, 373-383 (2014).

21. O. A. Ladyzhenskaya, The Mathematical Theory of Viscous Incompressible Flow, Gordon and Breach, New York (1969). 
22. O. A. Ladyzhenskaya and V. A. Solonnikov, "Some problems of vector analysis and generalized formulations of boundary-value problems for the Navier-Stokes equations," J. Math. Sci., New York 10, No. 2, 257-286 (1978).

23. J. Nečas, Direct Methods in the Theory of Elliptic Equations [in French], Masson et Cie, Paris; Academia, Prague (1967).

24. I. Babuška and A. K. Aziz, "Survey lectures on the mathematical foundations of the finite element method," In: The Mathematical Foundations of the Finite Element Method with Applications to Partial Differential Equations, pp. 5-359, Academic Press, New York (1972).

25. F. Brezzi, "On the existence, uniqueness and approximation of saddle-point problems arising from Lagrangian multipliers," Rev. Franc. Automat. Inform. Rech. Operat. 8, R-2, 129-151 (1974).

26. L. E. Payne and H. F. Weinberger, "An optimal Poincaré inequality for convex domains," Arch. Ration. Mech. Anal. 5, 286-292 (1960).

27. M. Costabel and M. Dauge, "On the inequalities of Babuška-Aziz, Friedrichs and HorganPayne," Arch. Ration. Mech. Anal. 217, No. 3, 873-898 (2015).

28. M. Dobrowolski, "On the LBB constant on stretched domains," Math. Nachr. 254-255, 64-67, (2003).

29. C. O. Horgan and L. E. Payne, "On inequalities of Korn, Friedrichs and Babuška-Aziz," Arch. Ration. Mech. Anal. 82, No. 2, 165-179 (1982).

30. M. A. Ol'shanskij and E. V. Chizhonkov, "On the best constant in the inf-sup condition for prolonged rectangular domains," Math. Notes 67, No. 3, 325-332 (2000).

31. M. A. Ol'shanskij and E. V. Chizhonkov, "On the domain geometry dependence of the LBB condition," Math. Model. Numer. Anal. 34, No. 5, 935-951 (2000).

32. L. E. Payne, "A bound for the optimal constant in an inequality of Ladyzhenskaya and Solonnikov," IMA J. Appl. Math. 72, No. 5, 563-569 (2007).

33. O. Mali, P. Neittaanmäki, and S. Repin, Accuracy Verification Methods. Theory and Algorithms, Springer, Dordrecht (2014).

34. O. A. Ladyzhenskaya and N. N. Ural'tseva, "Quasilinear elliptic equations and variational problems in several independent variables," Rus. Math. Surv. 16, No. 1, 17-91 (1961).

35. I. Ekeland and R. Temam, Convex Analysis and Variational Problems, North-Holland, New York, (1976).

36. S. Matculevich and S. Repin, "Explicit constants in Poincaré-type inequalities for simplicial domains and application to a posteriori estimates," Comput. Methods Appl. Math. 16, No. $2,277-298$ (2016).

37. A. I. Nazarov and S. I. Repin, "Exact constants in Poincaré type inequalities for functions with zero mean boundary traces," Math. Methods Appl. Sci. 38, No. 15, 3195-3207 (2015).

38. S. Repin, S. Sysala, and J. Haslinger, "Computable majorants of the limit load in Hencky's plasticity problems," Comput. Math. Appl. 75, No. 1, 199-217 (2018).

Submitted on April 8, 2019 